\title{
« Midi éternel ». Hommage de Dany Laferrière à Jacques Roumain
}

\section{Dany Laferrière}

\section{OpenEdition}

\section{Journals}

Édition électronique

URL : https://journals.openedition.org/coma/3198

DOI : $10.4000 /$ coma.3198

ISSN : 2275-1742

\section{Éditeur}

Institut des textes \& manuscrits modernes (ITEM)

\section{Référence électronique}

Dany Laferrière, « « Midi éternel ». Hommage de Dany Laferrière à Jacques Roumain », Continents manuscrits [En ligne], 11 | 2018, mis en ligne le 30 novembre 2018, consulté le 12 janvier 2023. URL : http://journals.openedition.org/coma/3198 ; DOI : https://doi.org/10.4000/coma.3198

Ce document a été généré automatiquement le 12 janvier 2023.

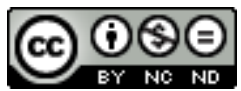

Creative Commons - Attribution - Pas d'Utilisation Commerciale - Pas de Modification 4.0 International - CC BY-NC-ND 4.0

https://creativecommons.org/licenses/by-nc-nd/4.0/ 


\title{
« Midi éternel ». Hommage de Dany Laferrière à Jacques Roumain
}

\author{
Dany Laferrière
}

\section{NOTE DE L'ÉDITEUR}

Lors de la présentation officielle de la réédition des Euvres complètes de Jacques Roumain, le 20 juin 2018, à l'École normale supérieure, l'écrivain Dany Laferrière, membre de l'Académie française, a prononcé quelques mots, dans lesquels il exprime sa grande proximité avec son prédécesseur. Il a autorisé la publication de la transcription de cette intervention. Qu'il en soit ici remercié.

1 C'est une vraie émotion que tenir Roumain dans ses mains, comme ça, tout Roumain... Je rêve! C'est le premier de notre littérature qui se présente ainsi en un volume. Dans cet exemplaire luxueux. En papier bible, on peut toucher Roumain. Je ressens comme un choc électrique. Et tout de suite me remonte à la mémoire ma première lecture de Roumain, comme si c'était hier. C'est cela un classique. L'émotion annule le temps. Une douce intemporalité se glisse en moi. L'impression qu'il m'attire à lui, et qu'on s'apprête à plonger ensemble dans les profondeurs. Je vois le visage grave presque granitique de l'auteur d'un véritable chef-d'œuvre, ce qui est plus rare qu'on ne pense : Gouverneurs de la rosée. On a envie de le retrouver à sa table de travail, dans ses doutes, ses angoisses et ses fêtes, au moment de l'écriture du roman. Ce qui me rappelle ce magnifique poème de Whitman où il aperçoit à la courbe d'un siècle son futur lecteur. Il raconte qu'au moment où il écrivait son poème le lecteur n'était pas encore né mais lui bien vivant, et maintenant que ce lecteur le lit il n'est plus. En fait ils sont tous les deux entremêlés dans une intemporalité qui s'appelle la littérature. Il y a des chefs-d'œuvre qui vous glace par leur perfection et d'autres comme Gouverneurs de la rosée qui vous prennent doucement la main pour une promenade dans leur univers. Je devrais écrire " paysage » dans le cas de Roumain car sa palette fait souvent penser à celle d'un de ces peintres primitifs. Le trait est pur, sobre. Pas de fioritures. Si ce n'était cette langue particulière, ce savoureux mélange de créole et de français du dix-huitième siècle, on 
ne ferait que regarder le paysage désolé, âpre, qui sert d'écrin à une histoire d'amour, malheureuse vers la fin, mais si gorgée de courage qu'on se demande si l'on peut aujourd'hui écrire un pareil roman en Haïti sans susciter le doute. Yanick Lahens l'a pourtant tenté et réussi. Ce qui fait que je reviens si souvent à ce roman, c'est cette complicité, je dirais mieux cette amitié que Roumain a su créer en 1968 avec un jeune lecteur de quinze ans. C'est l'âge rêvé pour lire ce roman qui vous accompagnera toute la vie.

2 Mais l'œuvre de Roumain ne s'arrête pas à son chef-d'œuvre. Gouverneurs de la rosée est la locomotive d'un train qui comporte des recueils de poèmes, des nouvelles amusantes ou poignantes, une correspondance qui montre que Roumain n'est pas Tartuffe, son envers ressemble à son endroit, des articles percutants dans les quotidiens de Port-auPrince et des essais politiques rageurs. Roumain a commencé sa carrière comme pamphlétaire. C'est un jeune homme de la classe bourgeoise dont la spécialité est dès le départ de tirer à boulets rouges sur les élites économiques et politiques, ce qui l'amène certaines fois en prison. Il peut paraître arrogant, violent, mais de son point de vue ce sont les exploiteurs qui sont vulgaires, grossiers et violents. Il est tumultueux, c'est sûr. Il n'hésite pas d'ailleurs à insulter un membre important de la famille de sa fiancée, à la veille de son mariage... le mariage a été interrompu, naturellement... il écrit dans le journal un article où il traite le président de la république (le parent en question) de tous les noms... il était tout jeune. Il écrivait sans cesse dans Le Nouvelliste. Il a commencé si tôt qu'on a dû lui donner une sorte de laissez-passer pour signer ses premiers textes. Je ne sais même pas si c'est lui qui signait ses chroniques au début. Il était si jeune... Roumain. On n'imagine pas quand on lit Gouverneurs de la rosée, ce texte d'une si grande impassibilité qu'on a l'impression que la narrateur était le village de Fonds-Rouge ${ }^{1}$ et non ses habitants. Je n'ai retrouvé une pareille impassibilité que dans Guerre et Paix de Tolstoï. Cette impression que les choses se déroulent sous les yeux de l'auteur presqu'en même temps que sous nos yeux... il y a quelque chose comme si le livre s'était écrit tout seul. Et pourtant on sent le Roumain bouillonnant parfois derrière certaines formules de solidarité, mais heureusement pas trop souvent. Il tenait la bride à ses convictions politiques, même si l'ensemble est un éloge mesuré du réalisme socialiste. Cela n'a rien à voir avec le roman tout aussi célèbre mais moins réussi de Jacques Stephen Alexis, Compère général Soleil. Justement Alexis n'a pas suivi la trace de son maître, il s'est trop énervé devant l'injustice et il a tenté trop souvent de sauter sur l'ennemi de classe.

3 Il y a une sorte de conjonction ces temps-ci, et qui annonce quelque chose de neuf et d'intéressant dans la fortune de la littérature haïtienne et qui permet son rayonnement sur le plan international. La littérature haïtienne est installée sur un trépied solide : Jacques Roumain, Jacques Stephen Alexis et Marie Vieux-Chauvet, trois personnages extrêmement importants qui occupent chacun un espace de la géographie littéraire du pays : Roumain a installé son laboratoire dans la paysannerie, à Fonds-Rouge ; Alexis s'est installé à Port-au-Prince, dans le combat ouvrier, il a même fondé un parti communiste - Roumain aussi d'ailleurs; et Vieux-Chauvet en province, dans la bourgeoisie traditionnelle, complètement corsetée, enfermée dans un univers passéiste, en pleine montée de la dictature de Duvalier. Ce trépied-là, ça tient, naturellement... ça peut tenir la chaudière haïtienne... Et la barre est haute pour tout jeune écrivain qui voudrait écrire en Haïti... on se sent littéralement cerné par cette triade. Si on essaie d'écrire un roman paysan, parce qu'on a passé son enfance en 
province, eh bien Gouverneurs de la rosée a depuis longtemps brûlé le paysage... il n'y a presqu'aucune possibilité. Dès qu'on met en scène, à la campagne, un narrateur qui revient de l'étranger, il ne peut que s'appeler Manuel. Le problème c'est que l'écrivain haïtien est un des rares types d'écrivains qui commence à écrire avant de lire ses prédécesseurs! Nous sommes tellement impétueux, nous voulons tellement dire notre monde, notre univers, nous dire. Et puis aussi, il y a cette lignée d'importants écrivains qui ressemblent à des totems qui intimident, paralysent même parfois, et dont Roumain est le chef de file.

Donc il est difficile d'échapper à Gouverneurs de la rosée. Cette tendresse. Un fleuve de lait selon Alexis. Le récit est d'une simplicité et d'une limpidité, ce midi éternel qui éclaire le roman. Tout tourne autour de cette nuit, pivotale, la nuit où Gervilien tue Manuel. Tout semblait perdu. Aucune espérance. Puis le Soleil, l'astre que Roumain appelle son compère et qui est pour Alexis un camarade. Cette lumière que je n'ai jamais réussi à épingler sur page. J'ai tenté mais en vain de décrire Port-au-Prince sous toutes les lumières possibles. Chaque pays à sa lumière, ici nous vivons sous deux dominations: la chaleur et la lumière. Il ne faut pas confondre la lumière avec la chaleur. Il arrive qu'au Québec, où je vis, la lumière éclatante du soleil n'entraîne aucune chaleur par moins quarante degrés. Ne sortez surtout pas dans la rue, même sous un soleil implacable. Cette lumière difficile à installer dans un roman, un tableau, une musique, Roumain l'a fait avec une telle force et une grâce si particulière. Et ça, il faut être du métier pour pouvoir apprécier ce travail... Une manière unique de placer les mots sur la page, comme s'il y avait deux étages. Ce qu'on dit au second étage n'est pas ce qui se passe au premier. On parle beaucoup du Roumain humaniste sans oublier qu'il a connu des jours difficiles avec la page blanche. Quand ses personnages vaquent à leurs occupations dans Fonds-Rouge on sent le soleil sur leur nuque. On sait, à tout moment, où se trouvent Manuel et Anaïse et on entend leur cœur battre près de la source.

5 Nous connaissons très bien l'autre compère, et c'est Jacques Stephen Alexis. Pourquoi je parle de ces trois larrons? D'abord parce que ce sont de grands écrivains mais surtout parce que Roumain adore le jeu collectif. Michaëlle Jean parlait tout à l'heure de coumbite. Roumain n'aurait jamais accepté d'être tout seul en avant. Il aurait bien aimé ça, un trio.

6 À sa mort... le texte se trouve dans ce livre magnifique de Jacques Stephen Alexis. Alexis, un tout jeune Alexis salue Roumain et annonce la germination prochaine. VieuxChauvet a un étrange destin aussi. Les Haïtiens ne peuvent pas faire les choses facilement. Il ne s'agit pas d'écrire, il faut avoir un destin. Roumain meurt jeune, à 37 ans. Alexis, lui, meurt dans les conditions qu'on connait : il rentre en Haïti, avec des amis, et se fait assassiner par les sbires de Duvalier ${ }^{2}$. Vieux-Chauvet meurt sans avoir vu le succès de son livre. Elle a vu son livre, mais pas sa gloire monter, parce qu'on avait acheté et pilonné l'édition complète de Amour, Colère et Folie. Trois destins dramatiques, comme ça. C'est un trépied "ardent», le mot a été prononcé tout à l'heure par Michaëlle Jean... ardent, c'est brûlant. On ne peut pas y toucher, il ne s'agit pas de mondanités littéraires. Les gens paient, ils paient de leur peau parfois, et ils paient pour tout le monde. Dernièrement Alexis a eu un prix littéraire, ah! En attendant, continuons sur Vieux-Chauvet - j'ai failli passer sur Vieux-Chauvet. Car Amour, Colère et Folie est aussi un chef-d'œuvre. On entend la rumeur qui claironne partout le succès phénoménal de ce roman qui déborde le cadre local. Vieux-Chauvet qui a dévoilé le 
style particulier de la dictature de Duvalier. C'est cette romancière bien installée dans la bourgeoisie haïtienne qui a écrit le grand roman des années noires du duvaliérisme. Elle s'est tout de suite retrouvée prise entre deux feux. Elle s'est retrouvée aux ÉtatsUnis et son livre pilonné. Son œuvre est toujours présente et elle brille d'une noire lumière. Gouverneurs de la rosée paru dans les années quarante a entrainé dans son sillage une pléiade d'écrivains qui ont installé leur laboratoire dans toutes les capitales du monde. On les croise à Dakar, à Berlin, à Paris, à Montréal, à New York, à Port-auPrince, à Bruxelles, à Rome... Ce sont les enfants de Roumain.

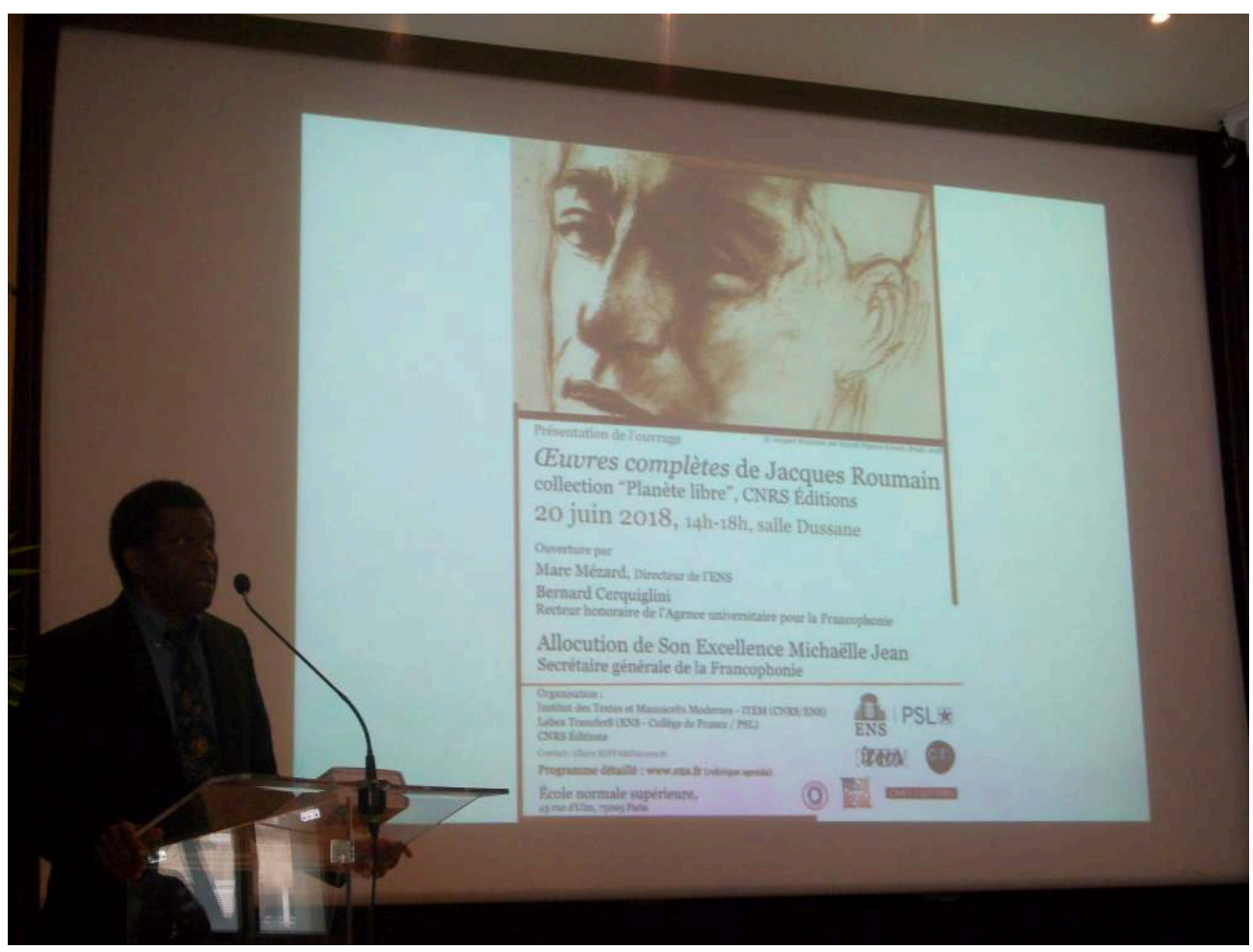

Dany Laferrière, 20 juin 2018, École normale supérieure, Paris.

Photo : Claire Riffard

\section{NOTES}

1. Le village dans lequel vivent les protagonistes de Gouverneurs de la rosée.

2. La mort de Jacques Stephen Alexis fut un des épisodes sanglants de la présidence de Duvalier : «En compagnie de quatre compagnons, Charles Adrien-Georges, Guy Béliard, Hubert DupuisNouillé et Max Monroe, il débarque sur la plage de Bombardopolis, avec probablement pour objectif de rallier le hounfort dédié aux loas racines des Alexis, Souvenance. Sans doute trahis, les membres de l'expédition furent arrêtés, torturés, exécutés. La mort de Jacques Stephen Alexis n'a jamais été officiellement reconnue » (http://ile-en-ile.org/alexis_jacques-stephen/, 15/08/2018). 
INDEX

Mots-clés : Jacques Roumain, Haïti, archives, Archivos, Planète libre, CNRS Éditions

\section{AUTEUR}

DANY LAFERRIÈRE

Écrivain, membre de l'Académie française 